\title{
The Cost of 2-Distinguishing Cartesian Powers
}

\author{
Debra L. Boutin \\ Department of Mathematics \\ Hamilton College, Clinton, NY 13323 \\ dboutin@hamilton.edu
}

Submitted: Jan 10, 2012; Accepted: Mar 21, 2013; Published: Mar 31, 2013

Mathematics Subject Classification: 05C25

\begin{abstract}
A graph $G$ is said to be 2-distinguishable if there is a labeling of the vertices with two labels so that only the trivial automorphism preserves the label classes. The minimum size of a label class in any such labeling of $G$ is called the cost of 2-distinguishing $G$ and is denoted by $\rho(G)$. The determining number of a graph $G$, denoted $\operatorname{Det}(G)$, is the minimum size of a set of vertices whose pointwise stabilizer is trivial. The main result of this paper is that if $G^{k}$ is a 2-distinguishable Cartesian power of a prime, connected graph $G$ on at least three vertices with $\operatorname{Det}(G) \leqslant k$ and $\max \{2, \operatorname{Det}(G)\}<\operatorname{Det}\left(G^{k}\right)$, then $\rho\left(G^{k}\right) \in\left\{\operatorname{Det}\left(G^{k}\right)\right.$, $\left.\operatorname{Det}\left(G^{k}\right)+1\right\}$. In particular, for $n \geqslant 3, \rho\left(K_{3}^{n}\right) \in\left\{\left\lceil\log _{3}(2 n+1)\right\rceil+1,\left\lceil\log _{3}(2 n+1)\right\rceil+2\right\}$.
\end{abstract}

\section{Introduction}

A labeling of the vertices of a graph $G$ with the integers $1, \ldots, d$ is called a $d$-distinguishing labeling if no non-trivial automorphism of $G$ preserves the label classes. A graph is called $d$-distinguishable if it has a $d$-distinguishing labeling. Albertson and Collins introduced distinguishing in [3]. Recent work shows that large members of many infinite graph families are 2-distinguishable. These families include hypercubes $Q_{n}$ with $n \geqslant 4$, Cartesian powers $K_{3}^{n}$ with $n \geqslant 3$, and $G^{n}$ for a connected graph $G \neq K_{2}, K_{3}$ and $n \geqslant 2$ [1, 4, 12, 13], Kneser graphs $K_{n: k}$ with $n \geqslant 6, k \geqslant 2$ [2], and (with seven small exceptions) 3-connected planar graphs [8]. In 2007 Wilfried Imrich posed the following question [11]: "What is the minimum number of vertices in a label class of a 2-distinguishing labeling for the hypercube $Q_{n}$ ?"

Call the minimum size of a label class in a 2-distinguishing labeling of a graph $G$ the cost of 2-distinguishing $G$ and denote it by $\rho(G)$. The 2-distinguishing labeling provided for $Q_{n}$ by Bogstad and Cowan in [4] shows that for $n \geqslant 4, \rho\left(Q_{n}\right) \leqslant n+2$. The best result known when Imrich originally asked the question was $\rho\left(Q_{n}\right) \approx \sqrt{n}[11]$. For $n \geqslant 5$ the author of the present paper showed that $\left\lceil\log _{2} n\right\rceil+1 \leqslant \rho\left(Q_{n}\right) \leqslant 2\left\lceil\log _{2} n\right\rceil-1[6]$. 
Recall that $Q_{n}$ is a Cartesian power of $K_{2}$. In this paper the question of the cost of 2-distinguishing is extended to Cartesian powers of other prime graphs.

A significant tool used in this work is the determining set [5], a set of vertices whose pointwise stabilizer is trivial. Albertson and Boutin showed in [2] that a graph is $d$ distinguishable if and only if it has a determining set that is $(d-1)$-distinguishable. (Set distinguishability will be defined in Section 2.3.) In particular, the complement of such a determining set is a label class in a $d$-distinguishing labeling of $G$. Thus, a graph is 2-distinguishable if and only if it has a determining set for which any automorphism that fixes it setwise must also fix it pointwise. In such a case, the determining set and its complement provide the two necessary label classes for a 2-distinguishing labeling. Thus in particular, the cost of 2-distinguishing a graph $G$ is bounded below by the size of a smallest determining set, $\operatorname{denoted} \operatorname{Det}(G)$.

This paper proves that if $G^{k}$ is a 2-distinguishable Cartesian power, with $G$ a prime connected graph on at least three vertices with $\operatorname{Det}(G) \leqslant k$ and $\max \{2, \operatorname{Det}(G)\}<$ $\operatorname{Det}\left(G^{k}\right)$, then $\rho\left(G^{k}\right) \in\left\{\operatorname{Det}\left(G^{k}\right)\right.$, $\left.\operatorname{Det}\left(G^{k}\right)+1\right\}$. (These hypotheses are mild and exclude relatively few graphs.) Note that in general the cost of 2-distinguishing and the size of a smallest determining set can be far apart. For example, work of Goff and Greenfield [9] (see Example 1 in Section 2.3) shows that the cost of 2-distinguishing a graph $G$ can be an arbitrarily large multiple of $\operatorname{Det}(G)$.

This paper is organized as follows. Definitions and facts about determining sets, Cartesian products, and distinguishing labelings are given in Section 2. This section formally sets out the key idea tying together determining sets and distinguishing labelings and provides important technical tools used in following sections. Section 3 states and proves the main theorem. Open questions are given in Section 4.

\section{Background}

Graphs throughout this paper will be assumed to be simple and finite.

\subsection{Determining Sets}

Let $G$ be a graph. A subset $\mathcal{A} \subseteq V(G)$ is said to be a determining set for $G$ if whenever $\varphi, \psi \in \operatorname{Aut}(G)$ so that $\varphi(x)=\psi(x)$ for all $x \in \mathcal{A}$, then $\varphi=\psi$. Thus every automorphism of $G$ is uniquely determined by its action on the vertices of a determining set. Every graph has a determining set since a set containing all but one vertex of the graph is determining. The determining number of $G$, denoted here by $\operatorname{Det}(G)$, is the minimum $r$ such that $G$ has a determining set of size $r$.

Recall that the setwise stabilizer of $\mathcal{A} \subseteq V(G)$ is the set of all $\varphi \in \operatorname{Aut}(G)$ for which $\varphi(x) \in \mathcal{A}$ for all $x \in \mathcal{A}$. In this case we say that $\mathcal{A}$ is invariant under $\varphi$, or $\varphi$ preserves $\mathcal{A}$, and we write $\varphi(\mathcal{A})=\mathcal{A}$. The pointwise stabilizer of $\mathcal{A}$ is the set of all $\varphi \in \operatorname{Aut}(G)$ for which $\varphi(x)=x$ for all $x \in \mathcal{A}$. It is easy to see [5] that $\mathcal{A}$ is a determining set for $G$ if and only if the pointwise stabilizer of $\mathcal{A}$ is trivial. 


\subsection{Automorphisms \& Characteristic Matrices}

Recall that the Cartesian product of graphs $G$ and $H$, denoted by $G \square H$, has vertex set $V(G) \times V(H)$ with an edge between vertices $(x, u)$ and $(y, v)$ if either $x$ is adjacent to $y$ in $G$ and $u=v$, or $u$ is adjacent to $v$ in $H$ and $x=y$. The Cartesian power $H^{k}$ is the Cartesian product of $H$ with itself $k$ times.

A good reference for Cartesian products is [10]. Recall that $H$ is prime with respect to the Cartesian product if it cannot be written as the Cartesian product of two smaller graphs. Every connected graph can be written uniquely (up to order) as the Cartesian product of prime factors, $H=G_{1} \square \cdots \square G_{m}$. Throughout this section assume that $H=G_{1} \square \cdots \square G_{m}$ is a prime decomposition of a connected graph $H$ with respect to the Cartesian product. (Note that a relatively prime decomposition would work as well.) Each vertex $U \in H$ can be written uniquely as a vector $U=\left(u_{1}, \ldots, u_{m}\right)$ with $u_{i} \in G_{i}$. Each automorphism of $H$ can be understood in the following way.

Theorem 1. [10] If $\varphi \in \operatorname{Aut}(H)$ then there is a permutation $\pi \in S_{m}$ and isomorphisms $\psi_{i}: G_{i} \rightarrow G_{\pi(i)}$ so that

$$
\varphi\left(u_{1}, \ldots, u_{m}\right)=\left(\psi_{\pi^{-1}(1)}\left(u_{\pi^{-1}(1)}\right), \ldots, \psi_{\pi^{-1}(m)}\left(u_{\pi^{-1}(m)}\right)\right) .
$$

Let $\mathcal{A}=\left\{U_{1}, \ldots, U_{r}\right\}$ be an ordered set of vertices of $H$. Let $M_{\mathcal{A}}$ be the $r \times m$ matrix whose $i^{\text {th }}$ row contains the coordinates for $U_{i}$ with respect to the given Cartesian product decomposition. Call $M_{\mathcal{A}}$ the characteristic matrix of $\mathcal{A}$. Note that the $j^{\text {th }}$ column of $M_{\mathcal{A}}$ consists of the $j^{\text {th }}$ coordinates of $U_{1}, \ldots, U_{r}$ and can be denoted $\left[\begin{array}{lll}U_{1, j} & \ldots & U_{r, j}\end{array}\right]^{T}$. We say the $j^{\text {th }}$ and $k^{\text {th }}$ columns of $M_{\mathcal{A}},\left[\begin{array}{lll}U_{1, j} & \ldots & U_{r, j}\end{array}\right]^{T}$ and $\left[\begin{array}{llll}U_{1, k} & \ldots & U_{r, k}\end{array}\right]^{T}$, are isomorphic images of each other if there exists an isomorphism $\psi: G_{j} \rightarrow G_{k}$ so that $\psi\left(U_{i, j}\right)=U_{i, k}$ for all $i$. We can now state criteria for a set to be a determining set of a Cartesian product as follows.

Lemma 1. [7] A set of vertices $\mathcal{A}$ is a determining set for $H$ if and only if each column of the characteristic matrix $M_{\mathcal{A}}$ contains a determining set for the appropriate factor of $H$ and no two columns of $M_{\mathcal{A}}$ are isomorphic images of each other.

\subsection{Distinguishing Labelings}

A labeling $f: V(G) \rightarrow\{1, \ldots, d\}$ is said to be $d$-distinguishing if only the trivial automorphism preserves the label classes. Every graph has a distinguishing labeling since each vertex can be assigned a distinct label. A graph is called d-distinguishable if it has a $d$-distinguishing labeling. If $G$ is a 2-distinguishable graph, call a label class in a 2distinguishing labeling of $G$ a distinguishing class. Define the cost of 2-distinguishing $G$ to be the minimum size of a distinguishing class in $G$ and denote it by $\rho(G)$.

We will also need to know what it means for a subset of vertices to be $d$-distinguishable. Let $\mathcal{A} \subseteq V(G)$. A labeling $f: \mathcal{A} \rightarrow\{1, \ldots, d\}$ is called $d$-distinguishing if whenever an automorphism fixes $\mathcal{A}$ setwise and preserves the label classes of $\mathcal{A}$ then it fixes $\mathcal{A}$ pointwise. Note that though such an automorphism fixes $\mathcal{A}$ pointwise, it is not necessarily trivial; it 
may permute vertices in the complement of $\mathcal{A}$. A set $\mathcal{A}$ is called d-distinguishable if it has a $d$-distinguishing labeling. By definition, $\mathcal{A}$ is 1-distinguishable if every automorphism that preserves $\mathcal{A}$ fixes it pointwise.

The following theorem and corollary tie together determining sets and distinguishing labelings and facilitate the work in this paper.

Theorem 2. [2] A graph is $d$-distinguishable if and only if it has a determining set that is $(d-1)$-distinguishable.

Corollary 2.1. Let $G$ be a 2-distinguishable graph. A subset $\mathcal{A} \subseteq V(G)$ is a distinguishing class for $G$ if and only if it is a determining set with the property that every automorphism that fixes it setwise, also fixes it pointwise. That is, $\mathcal{A}$ is distinguishing class if and only if each of the setwise stabilizer and the pointwise stabilizer for $\mathcal{A}$ is trivial.

In particular, suppose $\mathcal{A}$ is a 1-distinguishable determining set. The fact that it is 1-distinguishable means that any automorphism that preserves $\mathcal{A}$ as a set also fixes it pointwise. The fact that it is a determining set means that the only automorphism that fixes it pointwise is the trivial automorphism. Thus only the trivial automorphism fixes $\mathcal{A}$ setwise. If we label the vertices of $\mathcal{A}$ with 1 s and the vertices of its complement with $2 \mathrm{~s}$, only the trivial automorphism preserves the label classes. Therefore $\mathcal{A}$ is a distinguishing class of a 2-distinguishing labeling.

Thus in particular, the cost of 2-distinguishing a graph is bounded below by the size of a smallest determining set, $\operatorname{Det}(G)$. However, the fact that $\operatorname{Det}(G)$ is a lower bound does not mean that it is always close to the cost of 2-distinguishing. For instance, in the example below Goff and Greenfield [9] show that the cost of 2-distinguishing can be an arbitrarily large multiple of the determining number for a graph.

Example 1. Let $k$ be an integer greater than or equal to 3. Let $H$ be an asymmetric graph on $n=2 k$ vertices. Let $G$ be the disjoint union of $2^{n}$ copies of $H$. A set of vertices of $G$ is a determining set if and only if it contains at least one vertex from each copy of $H$. Thus $\operatorname{Det}(G)=2^{n}$. A labeling of $G$ with two labels is distinguishing if and only if each copy of $H$ gets a different labeling. There are $2^{n}$ distinct labelings of $H$ with two labels - exactly enough for one for each copy of $H$. Thus there is a unique (up to the order of the copies of $H$ ) 2-distinguishing labeling of $G$. Since all distinct labelings of $H$ with two labels occur, each label is used on exactly half the vertices. Thus $\rho(G)=k 2^{n}=k \operatorname{Det}(G)$.

\subsection{Tools for the Characteristic Matrix}

Let $\mathcal{A}=\left\{U_{1}, \ldots, U_{r}\right\} \subseteq V(H)$ and let $\varphi \in \operatorname{Aut}(H)$ so that $\varphi(\mathcal{A})=\mathcal{A}$. It will be useful to understand the effect of $\varphi$ on the characteristic matrix $M_{\mathcal{A}}$. Since $\varphi(\mathcal{A})=\mathcal{A}$, $M_{\varphi(\mathcal{A})}$ has the same rows as $M_{\mathcal{A}}$ but in a different order. That is, there is a permutation $\sigma \in S_{r}$ on the rows of $M_{\mathcal{A}}$ that yields $M_{\varphi(\mathcal{A})}$. However, since $\varphi \in \operatorname{Aut}(H)$ by Theorem 1 there is a permutation $\pi \in S_{m}$ on the columns of $M_{\mathcal{A}}$ and isomorphisms $\psi_{i}: G_{i} \rightarrow$ $G_{\pi(i)}$ so that the effect on $M_{\mathcal{A}}$ is to transform the $j^{\text {th }}$ column $\left[U_{1, j} \ldots U_{r, j}\right]^{T}$ of $M_{\mathcal{A}}$ to $\left[\psi_{\pi^{-1}(j)}\left(U_{1, \pi^{-1}(j)}\right) \ldots \psi_{\pi^{-j}(1)}\left(U_{r, \pi^{-1}(j)}\right)\right]^{T}$ in $M_{\varphi(\mathcal{A})}$. That is, the $j^{\text {th }}$ column in $M_{\varphi(\mathcal{A})}$ is an 
isomorphic image of the $\pi^{-1}(j)^{t h}$ column of $M_{\mathcal{A}}$. It is the interplay of the row permutation $\sigma$ and the column permutation $\pi$ together with the columns isomorphisms $\psi_{i}$ that yields the results in this paper.

Example 2. Let $U_{1}=10110101, U_{2}=11001100$ and $U_{3}=10001001$ be vertices of $Q_{8}=K_{2}^{8}$. The characteristic matrix of $\mathcal{A}=\left\{U_{1}, U_{2}, U_{3}\right\}$ is

$$
M_{\mathcal{A}}=\left[\begin{array}{llllllll}
1 & 0 & 1 & 1 & 0 & 1 & 0 & 1 \\
1 & 1 & 0 & 0 & 1 & 1 & 0 & 0 \\
1 & 0 & 0 & 0 & 1 & 0 & 0 & 1
\end{array}\right]
$$

Notice that columns 1 and 7 are isomorphic images of each other, as are columns 2 and 8 , and columns 3, 4, and 5. No column is an isomorphic image of column 6 . Consider $\varphi \in \operatorname{Aut}\left(Q_{8}\right)$ with corresponding factor (column) permutation $\pi=(28)$ and factor (column) isomorphisms $\psi_{2}, \psi_{8}$ equal to the non-trivial automorphism of $K_{2}$, while all other $\psi_{i}$ equal the trivial automorphism. Note that $M_{\varphi(\mathcal{A})}=M_{\mathcal{A}}$. Thus we see that $\varphi$ not only preserves $\mathcal{A}$ as a set, but fixes $\mathcal{A}$ pointwise.

Example 3. Let $U_{1}=0000, U_{2}=1010$ and $U_{3}=1100$ be vertices of $Q_{4}=K_{2}^{4}$. Let $\mathcal{A}=\left\{U_{1}, U_{2}, U_{3}\right\}$ and see $M_{\mathcal{A}}$ below. Note that no pair of columns are isomorphic images of each other and that each column contains a determining set for $K_{2}$. Thus by Lemma 1 , $\mathcal{A}$ is a determining set for $Q_{4}$. Consider $\varphi \in \operatorname{Aut}\left(Q_{4}\right)$ with corresponding factor (column) permutation $\pi=\left(\begin{array}{ll}1 & 3\end{array}\right)$ and factor (column) isomorphisms $\psi_{1}, \psi_{3}$ equal to the non-trivial automorphism of $K_{2}$, and $\pi_{2}, \pi_{4}$ equal to the trivial automorphism. See $M_{\varphi(\mathcal{A})}$ below. Note that we can obtain $M_{\varphi(\mathcal{A})}$ from $M_{\mathcal{A}}$ using the row permutation $\sigma=\left(\begin{array}{ll}1 & 2\end{array}\right)$. Thus $\sigma(\mathcal{A})=\mathcal{A}$ and more particularly, $\varphi\left(U_{1}\right)=U_{2}, \varphi\left(U_{2}\right)=U_{1}$, and $\varphi\left(U_{3}\right)=U_{3}$.

$$
M_{\mathcal{A}}=\left[\begin{array}{cccc}
0 & 0 & 0 & 0 \\
1 & 0 & 1 & 0 \\
1 & 1 & 0 & 0
\end{array}\right], \quad M_{\varphi(\mathcal{A})}=\left[\begin{array}{cccc}
1 & 0 & 1 & 0 \\
0 & 0 & 0 & 0 \\
1 & 1 & 0 & 0
\end{array}\right] .
$$

Given a characteristic matrix $M_{\mathcal{A}}$, classify each column by a set of positive integers $\left\{a_{1}, a_{2}, \ldots, a_{m}\right\}$ where $a_{1}$ is the largest number of positions of equal value in the column, $a_{2}$ is the second largest, et cetera. We will call $\left\{a_{1}, a_{2}, \ldots, a_{m}\right\}$ the column type. For instance, columns 1 and 7 of the characteristic matrix in Example 2 have type $\{3\}$, while the remaining columns have type $\{2,1\}$. Note that any two columns that are isomorphic images of each other must have the same column type, but that non-isomorphic columns may have the same type as well. Further note that any permutation of the rows of $M_{\mathcal{A}}$ leaves unchanged the number of positions of a given value in each column, and therefore preserves the column type of each column. Thus if $\varphi$ preserves the set $\mathcal{A}$, then $\sigma$ (the associated row permutation of $M_{\mathcal{A}}$ ) preserves the column type of each column. However, since under the action of $\varphi$, column $j$ of $M_{\varphi(\mathcal{A})}$ is an isomorphic copy of column $\pi^{-1}(j)$ of $M_{\mathcal{A}}$, and since column isomorphism preserves column type, the type of column $j$ of $M_{\varphi(\mathcal{A})}$ is the same as the type of column $\pi^{-1}(j)$ of $M_{\mathcal{A}}$. Thus for any $\varphi \in \operatorname{Aut}(H)$ the column permutation $\pi$ associated with $\varphi$ takes columns to columns of the same type. These facts will be important in proving the results in this paper. 
To use Lemma 1, it will be important to be able to construct characteristic matrices with the property that no pair of columns are isomorphic images of each other. To aid in this we will define a canonical way to choose a representative in each column orbit. Let $G$ be a factor of $H$. Assume a total order on the vertices of $G, u_{1} \leqslant u_{2} \leqslant$ $u_{3} \leqslant \cdots \leqslant u_{|V(G)|}$, and assume the induced lexicographic order on the (set) Cartesian product $G^{r}$. Let $C=\left[\begin{array}{llll}c_{1} & c_{2} & \cdots & c_{r}\end{array}\right]^{T}$ be a column ( $r$-vector) associated with the factor $G$ in a characteristic matrix. The set of $r$-vectors that are isomorphic to $C$ is $\mathcal{O}=$ $\left\{\psi(C)=\left[\psi\left(c_{1}\right) \psi\left(c_{2}\right) \cdots \psi\left(c_{r}\right)\right]^{T} \mid \psi \in \operatorname{Aut}(G)\right\}$. We will say $C$ is a lowest lexicographic representative, or $l-l-r$, if it is the least element (in the induced lexicographic order) in $\mathcal{O}$. That is, the vertex in the first position of $C$ is the least vertex in its orbit; the vertex in the second position is least over all second position vertices in columns isomorphic to $C$ which have this same lowest first position; the vertex in the third position is least over all third position vertices which have the same least first and second positions; etc. By definition, two l-l-r columns are isomorphic if and only if they are equal, and every column has an isomorphic image that is l-l-r. Note that we can build a column we know to be l-l-r by ensuring that $u_{2}$ occurs after the first occurrence of $u_{1}$, and $u_{3}$ occurs and the first occurrence of $u_{2}$, etc.

\section{Cost of 2-Distinguishing Cartesian Powers}

Theorem 3. If $G^{k}$ is a 2-distinguishable Cartesian power of a prime connected graph $G$ on at least three vertices with $\operatorname{Det}(G) \leqslant k$ and $\max \{2, \operatorname{Det}(G)\}<\operatorname{Det}\left(G^{k}\right)$, then $\rho\left(G^{k}\right) \in\left\{\operatorname{Det}\left(G^{k}\right), \operatorname{Det}\left(G^{k}\right)+1\right\}$.

Proof. Let $m=\max \{2, \operatorname{Det}(G)\}, r=\operatorname{Det}\left(G^{k}\right)$. Let $\ell_{r}$ be the largest integer so that $\operatorname{Det}\left(G^{\ell_{r}}\right)=r$. (Note that since $G$ is finite there are only a finite number of non-isomorphic columns of length $r$ and thus such an integer exists.) Let $T=\{1, \ldots, m\}$ be a determining set of size $m$ for $G$. Let $m+1, \ldots,|V(G)|$ denote the remaining vertices of $G$. Since we will be using vertices of $G$ as entries in a matrix, we will refer to $1,2, \ldots,|V(G)|$ as values rather than vertices. Assume the total order on the vertices of $G$ implied by the linear order of their values. (The order is necessary for the definition of l-l-r.) Let $\mathcal{U}=\left\{U_{1}, \ldots, U_{r}\right\}$ be a minimum size determining set for $G^{\ell_{r}}$. Let $N$ be the characteristic matrix for $\mathcal{U}$. Without loss of generality assume that each column of $N$ is l-l-r. (If some columns are not l-l-r, apply appropriate automorphisms of $G$ to them to make them l-l-r. Note that column isomorphism preserves the properties necessary for a characteristic matrix of a determining set given in Lemma 1 . Thus the rows of this modified matrix provide a minimum size determining set, say $\mathcal{U}^{\prime}$, for $G^{\ell_{r}}$. Further, since column isomorphisms correspond to automorphisms of $G^{\ell_{r}}, \mathcal{U}^{\prime}$ is an automorphic image of $\mathcal{U}$. Thus we may assume that $\mathcal{U}$ is such that all columns of $N$ are already l-l-r.) Since $\ell_{r}$ is maximal, $N$ contains all l-l-r columns of length $r$ that contain a determining set for $G$. (Otherwise we could add such a column, and get a determining set of size $r$ for $G^{\ell_{r}+1}$.)

First we will find a distinguishing class $\mathcal{A}$ for $G^{\ell_{r}}$ in separate cases depending on the values of $m$ and of $r$. Then we will modify $\mathcal{A}$ to a distinguishing class for $G^{k}$. 


$$
\left[\begin{array}{c}
U_{1} \\
U_{2} \\
U_{3} \\
U_{4} \\
U_{5} \\
U_{6} \\
U_{7} \\
U_{8} \\
U_{9} \\
X
\end{array}\right]=\left[\begin{array}{cccccccccc}
\underline{1} & \underline{1} & \underline{1} & 1 & 1 & 1 & 1 & 1 & 1 & \\
2 & \underline{2} & \underline{2} & \underline{2} & 1 & 1 & 1 & 1 & 2 & \\
2 & 3 & \underline{3} & \underline{3} & \underline{2} & 1 & 1 & 1 & 3 & \\
2 & 3 & 4 & \underline{4} & \underline{3} & \underline{2} & 1 & 1 & 4 & \\
2 & 3 & 4 & 1 & \underline{4} & \underline{3} & \underline{2} & 1 & 2 & \\
2 & 3 & 4 & 1 & 1 & \underline{4} & \underline{3} & \underline{2} & 2 & \\
2 & 3 & 4 & 1 & 1 & 1 & \underline{4} & \underline{3} & 2 & \\
\underline{3} & 3 & 4 & 1 & 1 & 1 & 1 & \underline{4} & 2 \\
\underline{4} & \underline{4} & 4 & 1 & 1 & 1 & 1 & 1 & 2 \\
2 & 3 & 4 & 1 & 1 & 1 & 1 & 1 & 1
\end{array}\right]
$$

Figure 1: First 9 columns when $r=9, m=4$. Singular values in each of first 8 columns are underlined.

Case 1: $m \geqslant 3$.

For $i \in\{1, \ldots, r-1\}$ define column $i$ to be the unique l-l-r column with values from $T$, with identical values in positions $i+1, \ldots, r, 1, \ldots, i-m+1$ (the $r-m+1$ consecutive positions modulo $r$ beginning at $i+1$ ), and with the remaining distinct values from $T$ in the remaining positions. Each of these columns has type $\{r-m+1,1, \ldots, 1\}$. Since $1<m<r$, we get that $1<r-m+1<r$ and therefore these columns are distinct. Define the $r^{\text {th }}$ column to be $\left[\begin{array}{llllllll}1 & 2 & 3 & \cdots & m & 2 & \cdots & 2\end{array}\right]^{T}$. This column is also l-l-r of type $\{r-m+1,1, \ldots, 1\}$, and since $m \geqslant 3$ this column is distinct from each of the first $r-1$ columns.

Since $N$ contains all l-l-r columns of length $r$ that contain a determining set for $G$, it must contain each of these $r$ columns. We can assume without loss of generality that the defined columns are in the described positions. (If not, permute the columns of $N$ until they do. Since this characteristic matrix still satisfies the conditions of Lemma 1, the rows of this modified matrix provide a minimum size determining set, say $\mathcal{U}^{\prime \prime}$, for $G^{\ell_{r}}$. Further, since column permutations correspond to automorphisms of $G^{\ell_{r}}, \mathcal{U}^{\prime \prime}$ is an automorphic image of $\mathcal{U}$. Thus we can assume $\mathcal{U}$ is such that $N$ has the defined columns in the described positions.) See Figure 1 for the first nine columns of $M_{\mathcal{A}}$ when $r=9$ and $m=4$.

In each of the first $r-1$ columns we will want to keep track of the singular values, that is, values that occur precisely once in the given column. Note that under column isomorphism, the value that occurs in a given position may change, but whether it is a singular value for its column does not. However, under row permutation, the positions in a given column that are singular may change, but the values that are singular will not. Both these facts will be important later. In Figure 1 the singular values are underlined in each of the first $r-1$ columns.

Add an $(r+1)^{s t}$ row to matrix $N$, creating an $(r+1) \times \ell_{r}$ matrix $M$, so that the first $r-1$ columns are precisely the columns of type $\{r-m+2,1, \ldots, 1\}$ in $M$ and the $r^{t h}$ column is the only column of type $\{r-m+1,2,1, \ldots, 1\}$ in $M$. Note that since $m<r$, these are distinct types. To accomplish this, for $i \in\{1, \ldots r-1\}$ append to column $i$ the 
value in position $i+1$. (This is the non-singular value for the column.) Append a 1 to column $r$. Append to the remaining $\ell_{r}-r$ columns a value that is an $(m+1)^{s t}$ value for that column. (We know that each of these columns must contain at least $\operatorname{Det}(G)=m$ distinct values. If a column contains $m+1$ or more distinct values, append one of these. If a column contains only $m$ distinct values, since $\operatorname{Det}(G)<|V(G)|$ for all $G$, there exists an $(m+1)^{s t}$ value. Append such a value to the column.) Since these rightmost $\ell_{r}-r$ columns contain at least $m+1$ distinct values, while the first $r$ columns contain precisely $m$, the column types of the first $r$ columns are different from the column types of any of the latter $\ell_{r}-r$.

Let the vertex of $G^{\ell_{r}}$ associated with row $r+1$ of $M$ be denoted $X$. Let $\mathcal{A}=\mathcal{U} \cup\{X\}$. Note that since $\mathcal{U}$ is a determining set for $G^{\ell_{r}}$, so is $\mathcal{A}$. By construction, $M=M_{\mathcal{A}}$ is the characteristic matrix for $\mathcal{A}$. We will see that $\mathcal{A}$ is a distinguishing class for $G^{\ell_{r}}$.

Let $\varphi \in \operatorname{Aut}\left(G^{\ell_{r}}\right)$ so that $\varphi(\mathcal{A})=\mathcal{A}$. As discussed earlier, $\varphi$ acts as a permutation $\sigma$ on the rows of $M_{\mathcal{A}}$. But since $\varphi \in \operatorname{Aut}\left(G^{\ell_{r}}\right), \varphi$ also acts as a permutation $\pi$ of the columns of $M_{\mathcal{A}}$ composed with isomorphisms $\psi_{i}$ of individual columns.

By construction, the first $r-1$ columns are the only columns of $M$ of type $\{r-m+$ $2,1,1, \ldots, 1\}$ and the $r^{\text {th }}$ column is the only one of its type. As argued in Section 2.2, since $\varphi(\mathcal{A})=\mathcal{A}$, the column permutation $\pi$ can only permute columns of the same type. Thus the set of columns $\{1, \ldots, r-1\}$ is invariant under $\pi$, and $\pi(r)=r$.

Claim: $\varphi(X)=X$

Recall that by construction, each of the first $r-1$ columns has type $\{r-m+2,1, \ldots, 1\}$ and therefore contains $m-1 \geqslant 2$ singular values. In particular, for $i \in\{1, \ldots, r-1\}$, column $i$ has a singular value in positions $i-1$ and $i$ of $M_{\mathcal{A}}$. Thus each of the rows $1, \ldots, r$ contains at one least value in its first $r-1$ coordinates that is a singular value for its column. Thus, the $(r+1)^{s t}$ row is the only row of $M_{\mathcal{A}}$ for which each of the first $r-1$ coordinates contains a value that is non-singular in its column. Since the first $r-1$ columns are invariant under $\pi$, and the property of having the last position in a column equal to values in other positions is preserved under column isomorphism, the same is true in $M_{\varphi(\mathcal{A})}$. Thus the $(r+1)^{s t}$ row of $M_{\varphi(\mathcal{A})}$ (the $\sigma^{-1}(r+1)^{s t}$ row of $\left.M_{\mathcal{A}}\right)$ is the only row for which each of the first $r-1$ coordinates contains a value that is non-singular in its column. Thus $\sigma^{-1}(r+1)=r+1$ and $\varphi(X)=X$.

Claim: $\varphi\left(U_{1}\right)=U_{1}$.

Recall that $\pi(r)=r$ and that $\sigma(r+1)=r+1$. Further, in $M_{\mathcal{A}}$ only the first position in the $r^{\text {th }}$ column matches the $(r+1)^{s t}$ position. Since $\pi(r)=r$, and column isomorphism preserves which positions in a given column match, the same is true in $M_{\varphi(\mathcal{A})}$. Thus the $r^{\text {th }}$ coordinate of the first row of $M_{\varphi(\mathcal{A})}$ (row $\sigma^{-1}(1)$ of $M_{\mathcal{A}}$ ) must match the $r^{\text {th }}$ coordinate the $\sigma(r+1)=(r+1)^{s t}$ row. But the first row of $M_{\mathcal{A}}$ is the only one whose $r^{t h}$ coordinate matches that of the $(r+1)^{s t}$ row. Thus $\sigma^{-1}(1)=1$ and $\varphi\left(U_{1}\right)=U_{1}$.

Claim: $\varphi$ fixes each of $U_{2}, \ldots, U_{r-m+1}$.

Note that the first $r-m+1$ rows have precisely $m-1$ of their first $r-1$ coordinates containing values that are singular in their columns. The next $m-1$ rows have only $m-2$ of their first $r-1$ coordinates containing values that are singular in their columns. Since 
$\pi$ leaves the first $r-1$ columns invariant, and since column isomorphisms preserve the the positions in which singular values occur in columns, the number per row of singular values in the first $r-1$ coordinates is invariant under $\varphi$. Thus, the first $r-m+1$ rows are invariant under $\sigma$ as are the next $m-1$ rows.

Since row 1 of $M_{\mathcal{A}}$ has singular values in coordinates $1, \ldots, m-1$, row permutation maintains which values are singular values, we know that row 1 of $M_{\varphi(\mathcal{A})}$ has singular values in the same coordinates as row $\sigma^{-1}(1)=1$. That is, it has singular values in coordinates $1, \ldots, m-1$. Since column isomorphism preserves the positions in a column that contain singular values, and only columns $1, \ldots m-1$ have singular values in their first position, $\pi(\{1, \ldots, m-1\})=\{1, \ldots, m-1\}$. Suppose that $\sigma^{-1}(2)=j \in\{1, \ldots, r-$ $m+1\}$. As argued above, row 2 of $M_{\varphi(\mathcal{A})}$ has singular values in the same coordinates as row $\sigma^{-1}(2)=j$ of $M_{\mathcal{A}}$. These coordinates are $j, \ldots, m+j-2$ which implies that $\pi(\{2, \ldots, m\})=\{j, \ldots, m+j-2\}$. Since $\pi$ is a permutation this means that $\pi(\{1, \ldots, m-$ $1\} \cap\{2, \ldots, m\})=\pi(\{2, \ldots, m-1\})=\{1, \ldots, m-1\} \cap\{j, \ldots, m+j-2\}$ has size $m-2$. Since each is a set of consecutive integers, this is true if and only if $j=2$. Thus $\sigma(2)=2$. Continuing this process, we can conclude that $\sigma(i)=i$ for all $i \in\{1, \ldots, r-m+1\}$. Thus $\varphi$ fixes each of $U_{2}, \ldots, U_{r-m+1}$.

Claim: $\varphi$ fixes $U_{r-m+2}, \ldots, U_{r}$.

Note that since $\sigma$ fixes the first $r-m+1$ rows, column 1 contains a 1 followed by $r-m$ $2 \mathrm{~s}$. Recall that row permutations preserves which values are singular and which are nonsingular in a given column. Thus in column 1,1 is a singular value and 2 is a non-singular value. Since column 1 of $M_{\mathcal{A}}$ is the only of the first $r-1$ columns to have a singular value in its first position and a non-singular value in its second, and these properties are preserved by column permutation/isomorphism, $\pi(1)=1$. Since the $(r-m+2)^{s t}$ position of column 1 of $M_{\varphi(\mathcal{A})}$ (and $M_{\mathcal{A}}$ ) is a non-singular value, row $r-m+2$ of $M_{\varphi(\mathcal{A})}$ begins with a value that is non-singular for it's column. However, the $(r-m+2)^{n d}$ row of $M_{\mathcal{A}}$ is unique among rows $r-m+2, \ldots, r$ of $M_{\mathcal{A}}$ in having a non-singular value in its first coordinate. Thus $\sigma(r-m+2)=r-m+2$. We can now argue as we did above that $\sigma$ preserves $r-m+2, \ldots, r$. Thus $\varphi$ fixes $U_{r-m+2}, \ldots, U_{r}$. Thus $\varphi$ fixes all the vertices in $\mathcal{A}$. Thus $\mathcal{A}$ is a distinguishing class for $G^{\ell_{r}}$.

Case 2: $m=2$ and $r \geqslant 5$.

This proof strongly resembles the proof for $m \geqslant 3$. Recall our setup: $r=\operatorname{Det}\left(G^{k}\right) ; \ell_{r}$ is the largest integer so that $G^{\ell_{r}}$ has a minimum size determining set of size $r ; T=\{1,2\}$ is a determining set for $G ; \mathcal{U}$ is a minimum size determining set for $G^{\ell_{r}} ; N$ is the characteristic matrix for $\mathcal{U}$; and all columns of $N$ are l-l-r.

For $i \in\{1, \ldots, r-1\}$ define column $i$ to be the unique l-l-r column with values from $T$, with identical values in positions $i$ and $i+1$. Thus each of these columns has type $\{r-2,2\}$. Note that since $r \geqslant 5$ these columns are all distinct. Define the $r^{\text {th }}$ column to be $\left[\begin{array}{llll}1 & 2 & \cdots & 2\end{array}\right]^{T}$, which is l-l-r of type $\{r-1,1\}$. Since $\ell_{r}$ is the maximum integer so that $G^{\ell_{r}}$ has a determining set of size $r, N$ contains all non-isomorphic l-l-r columns of length $r$ and thus it contains the described columns. We can assume these columns arise in the described positions or choose an automorphic image of $\mathcal{U}$ for which it is true. 


$$
\left[\begin{array}{c}
U_{1} \\
U_{2} \\
U_{3} \\
U_{4} \\
U_{5} \\
U_{6} \\
U_{7} \\
U_{8} \\
U_{9} \\
X
\end{array}\right]=\left[\begin{array}{cccccccccc}
\underline{1} & 1 & 1 & 1 & 1 & 1 & 1 & 1 & 1 & \\
\underline{1} & \underline{2} & 1 & 1 & 1 & 1 & 1 & 1 & 2 & \\
2 & \underline{2} & \underline{2} & 1 & 1 & 1 & 1 & 1 & 2 & \\
2 & 1 & \underline{2} & \underline{2} & 1 & 1 & 1 & 1 & 2 & \\
2 & 1 & 1 & \underline{2} & \underline{2} & 1 & 1 & 1 & 2 & \\
2 & 1 & 1 & 1 & \underline{2} & \underline{2} & 1 & 1 & 2 & \\
2 & 1 & 1 & 1 & 1 & \underline{2} & \underline{2} & 1 & 2 & \\
2 & 1 & 1 & 1 & 1 & 1 & \underline{2} & \underline{2} & 2 \\
2 & 1 & 1 & 1 & 1 & 1 & 1 & \underline{2} & 2 \\
2 & 1 & 1 & 1 & 1 & 1 & 1 & 1 & 2
\end{array}\right]
$$

Figure 2: First 9 columns of $M_{A}$ when $r=9$. Duplicate values in each column are underlined.

Denote the vertex associated with row $i$ of $N$ by $U_{i}$. Then $\mathcal{U}=\left\{U_{1}, \ldots, U_{r}\right\}$ and $N=M_{\mathcal{U}}$. Add an $(r+1)^{s t}$ row to the matrix $N$, creating a matrix $M$, so that the first $r-1$ columns are precisely the columns of type $\{r-1,2\}$ in $M$ and the $r^{t h}$ column is the only one of type $\{r, 1\}$. (Since $r \neq 2$, these are distinct types.) We accomplish this by appending to column $i$ for $i \in\{1, \ldots r-1\}$ the value not in position $i$ or $i+1$, appending 2 to column $r$, and for columns outside the first $r$, appending a third value to the column. (If a column already contains three or more values, it doesn't matter which value we choose to append in position $r+1$. If a column contains only two distinct values, append a third. Since we assumed $G$ had at least three vertices, this is possible.) Let the vertex of $G^{\ell_{r}}$ associated with the $(r+1)^{s t}$ row be denoted $X$. Let $\mathcal{A}=\mathcal{U} \cup\{X\}$. Then $M=M_{\mathcal{A}}$. See Figure 2 to see the first eight columns of $M$ when $r=9$. We will see that $\mathcal{A}$ is a distinguishing class for $G^{\ell_{r}}$.

Let $\varphi \in \operatorname{Aut}\left(G^{\ell_{r}}\right)$ so that $\varphi(\mathcal{A})=\mathcal{A}$. As discussed earlier, since $\varphi(\mathcal{A})=\mathcal{A}, \varphi$ acts as a permutation $\sigma$ on the rows of $M_{\mathcal{A}}$ as well as a permutation $\pi$ of the columns of $M_{\mathcal{A}}$ composed with isomorphisms $\psi_{i}$ of individual columns. Since column type is invariant under column isomorphism, the set $\{1, \ldots, r-1\}$ is invariant under $\pi$, and $\pi(r)=r$.

Since $\pi(r)=r$, and since the $r^{\text {th }}$ column of $M_{\mathcal{A}}$ has a singular value in its first position, and since the property of a position containing a singular value is invariant under column isomorphism, the $r^{\text {th }}$ column of $M_{\varphi(\mathcal{A})}$ has a singular value in its first position as well. But since $\varphi$ acts as a row permutation $\sigma$ on $M_{\mathcal{A}}$, the first row of $M_{\varphi(\mathcal{A})}$ (the $\sigma^{-1}(1)^{s t}$ row of $M_{\mathcal{A}}$ ) must have as its $r^{\text {th }}$ coordinate a value that is singular in its column. But row 1 of $M_{\mathcal{A}}$ is the only such row with this property. Thus $\sigma(1)=1$.

Since $\sigma(1)=1$, the first column of $M_{\varphi(\mathcal{A})}$ must contain a duplicate value in position 1. Since the only column of $M_{\mathcal{A}}$ for which this is true is the first column, we have that $\pi(1)=1$. Then the second row of $M_{\varphi(\mathcal{A})}$ must have a duplicate value in its second coordinate; thus so does the $\sigma^{-1}(2)^{n d}$ row of $M_{\mathcal{A}}$. However the only row of $M_{\mathcal{A}}$ with a duplicate value in its second position, is its second. Thus $\sigma(2)=2$. Continuing in this manner we see that $\sigma$ fixes the rows $1, \ldots, r$ of $M_{\mathcal{A}}$. Since $\sigma \in S_{r+1}$ fixes each of $1, \ldots, r$, it must also fix $r+1$. Thus $\sigma$ fixes all the rows of $M_{\mathcal{A}}$ which tells us that $\varphi$ fixes all the 
vertices in $\mathcal{A}$. Thus $\mathcal{A}$ is a distinguishing class for $G^{\ell_{r}}$.

Case 3: $m=2$ and $r \leqslant 4$.

Consider the set of vertices of $\mathcal{A}=\left\{U_{1}, \ldots, U_{r}, X\right\} \subset V\left(G^{\ell_{r}}\right)$ whose characteristic matrix has the following first $r$ columns and whose remaining columns are distinct l-l-r columns containing a determining set for $G$ and containing at least three distinct values. By Lemma $1, \mathcal{A}$ is a determining set for $G^{\ell_{r}}$. In arguments analogous to those above, it can be shown that $\mathcal{A}$ is a distinguishing class for $G^{\ell_{r}}$.

$$
\begin{aligned}
& \text { For } r=4, M_{\mathcal{A}}=\left[\begin{array}{c}
U_{1} \\
U_{2} \\
U_{3} \\
U_{4} \\
X
\end{array}\right]=\left[\begin{array}{lllll}
1 & 1 & 1 & 1 \\
1 & 2 & 1 & 2 \\
2 & 2 & 2 & 2 & \ldots \\
2 & 1 & 2 & 2 \\
2 & 1 & 1 & 2
\end{array}\right] . \\
& \text { For } r=3, M_{\mathcal{A}}=\left[\begin{array}{c}
U_{1} \\
U_{2} \\
U_{3} \\
X
\end{array}\right]=\left[\begin{array}{llll}
1 & 1 & 1 & \\
2 & 2 & 2 & \\
2 & 1 & 1 & \ldots \\
2 & 1 & 2 &
\end{array}\right] .
\end{aligned}
$$

Claim: $\mathcal{A}$ projects to a distinguishing class for $G^{k}$.

Suppose that $\ell_{r-1}<k<\ell_{r}$. Let $p_{k}: G^{\ell_{r}} \rightarrow G^{k}$ be the standard projection map that deletes the final $\ell_{r}-k$ components of vertices of $G^{\ell_{r}}$. Let $\mathcal{A}$ be defined as above and denote $\left\{p_{k}\left(U_{1}\right), \ldots, p_{k}\left(U_{r}\right), p_{k}(X)\right\}$ by $p_{k}(\mathcal{A})$. Let $\varphi$ be in the setwise stabilizer of $p_{k}(\mathcal{A})$. Let $M_{p_{k}(\mathcal{A})}$ and $M_{\varphi\left(p_{k}(\mathcal{A})\right)}$ be the appropriate characteristic matrices.

The argument proving that $\mathcal{A}$ is a distinguishing class for $G^{\ell_{r}}$ used only the first $r$ columns of $\mathcal{A}$. Lemma 2, which follows the proof of this theorem and its corollary, proves that $r \leqslant k$. Thus the first $r$ columns of $M_{\mathcal{A}}$ are the same as the first $r$ columns of $M_{p_{k}(\mathcal{A})}$. Thus if $\varphi$ fixes $p_{k}(\mathcal{A})$ setwise, it must also fix it pointwise. Therefore $p_{k}(\mathcal{A})$ is a distinguishing class for $G^{k}$ of size $r+1$.

Corollary 3.1. For $n \geqslant 3,\left\lceil\log _{3}(2 n+1)\right\rceil+1 \leqslant \rho\left(K_{3}^{n}\right) \leqslant\left\lceil\log _{3}(2 n+1)\right\rceil+2$.

Proof. This uses the fact that $\operatorname{Det}\left(K_{3}^{n}\right)=\left\lceil\log _{3}(2 n+1)\right\rceil+1$ from [7], and that for $n \geqslant 3$, $K_{3}^{n}$ is 2-distinguishable [13].

Lemma 2. If $G$ is a print connected graph and $k$ a positive integer so that $\operatorname{Det}(G) \leqslant k$ and $\operatorname{Det}(G)<\operatorname{Det}\left(G^{k}\right)$ then $\operatorname{Det}\left(G^{k}\right) \leqslant k$.

Proof. Note that since $\operatorname{Det}(G)<\operatorname{Det}\left(G^{k}\right), 2 \leqslant k$. Let $m=\max \{2, \operatorname{Det}(G)\}$. Thus with $m$ as defined we have $m \leqslant k$. Let $T=\{1, \ldots, m\}$ be a determining set for $G$. (If $m=\operatorname{Det}(G)$, this will be a minimum size determining set. If $\operatorname{Det}(G)=1$, then $|V(G)| \geqslant 2$ and we can add a vertex to a minimum size determining set to get a determining set of size two.) Create an $k \times k$ matrix so that column $i$ has the value $i$ in each of the positions $i$ through $i+k-m$ modulo $k$ and values $1, \ldots, i-1, i+1, \ldots, m$ in the remaining positions. Note that since $2 \leqslant m \leqslant k$, we have that $1 \leqslant k-m+1 \leqslant k-1$ and each column has its repeated values in distinct positions. Since the position of repeated 
values is invariant under column isomorphism, no pair of columns is isomorphic. Since in addition, each column is constructed to contain a determining set for $G$, by Lemma 1, this is a characteristic matrix for a determining set of size $k$ for $G^{k}$. Thus $\operatorname{Det}\left(G^{k}\right) \leqslant k$.

Note that an important tool in this work was the fact that $G$ had more than $m=$ $\max \{2, \operatorname{Det}(G)\}$ vertices. This allowed us to force the column types of columns $r+1, \ldots, \ell_{r}$ to be distinct from the column types of the first $r$ columns, and therefore to guarantee that the first $r$ columns were invariant under any automorphism that preserved $\mathcal{A}$. This tool does not exist for $Q_{n}=K_{2}^{n}$. Thus the techniques in this paper cannot be immediately used to get analogous results for $Q_{n}$.

\section{Open Questions}

Question 1. Classify 2-distinguishable Cartesian powers $G^{k}$ so that $\rho\left(G^{k}\right)=\operatorname{Det}\left(G^{k}\right)$ (or so that $\rho\left(G^{k}\right)=\operatorname{Det}\left(G^{k}\right)+1$ ).

Question 2. Find more 2-distinguishable graph families $\mathcal{G}$ with the property that for all $G \in \mathcal{G}, \rho(G) \in\{\operatorname{Det}(G), \operatorname{Det}(G)+1\}$.

Question 3. Find 2-distinguishable graph families $\mathcal{G}$ with the property that for all $G \in \mathcal{G}$, $\rho(G)=\operatorname{Det}(G)$ (or so that $\rho(G)=\operatorname{Det}(G)+1$ ).

Question 4. Find minimal determining sets for families of 2-distinguishable graphs to help find minimum distinguishing classes.

\section{References}

[1] Michael O. Albertson. Distinguishing Cartesian powers of graphs. Electron. J. Combin., 12:Note 17 (electronic), 2005.

[2] Michael O. Albertson and Debra L. Boutin. Using determining sets to distinguish Kneser graphs. Electron. J. Combin., 14(1):Research Paper 20 (electronic), 2007.

[3] Michael O. Albertson and Karen L. Collins. Symmetry breaking in graphs. Electron. J. Combin., 3(1):Research Paper 18 (electronic), 1996.

[4] Bill Bogstad and Lenore J. Cowen. The distinguishing number of the hypercube. Discrete Math., 283(1-3):29-35, 2004.

[5] Debra L. Boutin. Identifying graph automorphisms using determining sets. Electron. J. Combin., 13(1):Research Paper 78 (electronic), 2006.

[6] Debra L. Boutin. Small label classes in 2-distinguishing labelings. Ars Math. Contemp., 1(2):154-165, 2008.

[7] Debra L. Boutin. The determining number of a Cartesian product. J. Graph Theory, 61(2):77-87, 2009. 
[8] Tomotaka Fukuda, Seiya Negami, and Thomas W. Tucker. 3-connected planar graphs are 2-distinguishable with few exceptions, Yokohama Math. J., 54(2):143-153, 2008.

[9] Michael Goff and Kara Greenfield. Personal communication.

[10] Richard Hammack, Wilfried Imrich, and Sandi Klavžar. Handbook of product graphs. Discrete Mathematics and its Applications (Boca Raton). CRC Press, Boca Raton, FL, second edition, 2011.

[11] Wilfried Imrich. Personal communication.

[12] Wilfried Imrich and Sandi Klavžar. Distinguishing Cartesian powers of graphs. J. Graph Theory, 53(3):250-260, 2006.

[13] Sandi Klavžar and Xuding Zhu. Cartesian powers of graphs can be distinguished by two labels. European J. Combin., 28(1):303-310, 2007. 